drug use (IDU), sex with a person who injected drugs (PWID), and methamphetamine use. DIS interviewed eleven $(69 \%)$ of the women following HIV diagnosis; nine $(82 \%)$ reported at least one of following: IDU, methamphetamine use, exchange sex, or serodiscordant, bisexual, or PWID sex partners.

Conclusion HIV incidence among King County women with gonorrhea and syphilis appears to be low. Although these diagnoses do not clearly identify a population that would benefit from the draft PrEP recommendations, the generalizability of these findings is uncertain and additional data on HIV incidence among women following bacterial STIs are needed.

Disclosure No significant relationships.

\section{P434 SUBSTANCE USE PATTERNS AND HIV PREVENTION AMONG GAY AND BISEXUAL MEN IN THE 2014-2015 SEX NOW SURVEY}

${ }^{1}$ Kiffer Card*, ${ }^{2}$ Robert Higgins, ${ }^{2}$ Len Tooley, ${ }^{2}$ Aidan Ablona, ${ }^{2}$ Terry Trussler, ${ }^{2}$ Jody Jollimore, ${ }^{1}$ Nathan Lachowsky. 'University of Victoria, School of Public Health and Social Policy, Victoria, Canada; ${ }^{2}$ Community Based Research Centre, Vancouver, Canada

\subsection{6/sextrans-2019-sti.520}

Background Qualitative studies suggest that substance use is central to the identities and cultures of many gay and bisexual men (gbMSM) - and a salient factor in how they manage HIV risk. To quantitatively assess this, we examined patterns of substance use and associations with awareness, interest, and uptake of key prevention strategies.

Methods Canadian gbMSM were recruited online and asked to report their frequency of substance use. Latent class analysis identified patterns in use. Demographic-adjusted multivariable multinomial logistic regression models, stratified by HIVstatus, assessed associations with key prevention strategies (TasP awareness, PrEP interest, HIV-testing).

Results Among 669 HIV-positive and 7,184 HIV-negative men, six substance use classes were characterized: 'limited' (46.0\%; i.e., infrequent/low use of most drugs), 'conventional' (25.9\%; i.e., alcohol, marijuana, and tobacco), 'club' (9.5\%; i.e., alcohol, cocaine, and psychedelics), 'sex' (11.4\%; i.e., alcohol, crystal meth, GHB, poppers, and erectile dysfunction drugs), 'prescription' (12.1\%; i.e., alcohol and prescription drugs), and 'assorted' (4.5\%; i.e., most drugs) use. Limited use was selected as the referent class in all analyses. Other HIV-positive men were no more likely to have detectable viral-loads, nor were they less likely to know about the preventative benefits of TasP. HIV-negative men in the prescription (aOR:1.37,95\%CI:1.15-1.63) and sex (aOR:1.58,95\%CI:1.212.06) drug use classes were more likely to know about TasP. HIV-negative men in the prescription (aOR:1.6, 95\%CI:1.341.91), conventional (aOR:1.30,95\% CI:1.16-1.45), club (aOR:1.44,95\%CI:1.15-1.81), sex (aOR:3.94,95\%CI:2.925.33), and assorted (aOR:3.06,95\%CI:1.64-5.72) use classes were more likely to report interest in taking PrEP. Membership in these classes was associated with higher odds of HIVtesting.

Conclusion Among HIV-positive men, we observed high levels of viral-load undetectability and TasP awareness, independent of substance use. Among HIV-negative men, multiple patterns of substance use traditionally associated with heightened risk for acquiring HIV were associated with awareness, interest, and uptake of HIV risk management strategies, contravening stereotypes that link substance use to risk-indifferent attitudes. Disclosure No significant relationships.

\section{P435 USE OF CONDOMS FOR THE PREVENTION OF SEXUALLY TRANSMITTED INFECTIONS (STIS) AMONG HIV PRE- EXPOSURE PROPHYLAXIS (PREP) USERS}

${ }^{1}$ Benjamin Bavinton, ${ }^{2}$ Stefanie Vaccher, ${ }^{3}$ Martin Holt, ${ }^{4}$ Rebecca Guy, ${ }^{1}$ Garrett Prestage, ${ }^{2}$ Fengyi Jin, ${ }^{5}$ Janaki Amin, ${ }^{6}$ David Templeton, ${ }^{7}$ Christine Selvey, ${ }^{8}$ Iryna Zablotska-Manos, ${ }^{9}$ Josephine Lusk, ${ }^{2}$ Erin Ogilvie, ${ }^{2}$ Tobias Vickers, ${ }^{10}$ Don Smith, ${ }^{11}$ Andrew Grulich. ${ }^{1}$ UNSW Sydney, The Kirby Institute, Sydney, Australia; ${ }^{2}$ The Kirby Institute, UNSW Sydney, Sydney, Australia; ${ }^{3}$ UNSW Sydney, Centre for Social Research in Health, Sydney, Australia; ${ }^{4}$ Kirby institute, Sydney, Australia; ${ }^{5}$ Macquarie University, Sydney, Australia; ${ }^{6}$ RPA Sexual Health, Sydney, Australia; ${ }^{7}$ NSW Ministry of Health, Sydney, Australia; ${ }^{8}$ Sydney Medical School Westmead, University of Sydney, Sydney, Australia; ${ }^{9}$ Short Street Centre, Sydney, Australia; ${ }^{10}$ Albion Centre, Sydney, Australia; ${ }^{11}$ Kirby Institute, the University of New South Wales, Sydney, Australia

\subsection{6/sextrans-2019-sti.521}

Background HIV PrEP is highly effective at preventing HIV, but not STI, acquisition. We examined the use of condoms to prevent STIs among PrEP-users in the EPIC-NSW trial.

Methods Between March 2016 and April 2018, 9,708 individuals were enrolled. Analysis was restricted to participants who completed the optional end-of-study survey and were on-study for $\geq 1$ year prior. STI diagnoses came from medical records; other data were from the end-of-study survey. We used multivariate logistic regression to assess independent associations between those agreeing with the statement, "I use condoms to avoid getting STIs" ("STI-condom-users") and those disagreeing. Results 2,915 participants were included; $92.5 \%$ were gay men. In the previous year, 54.2\%, 25.6\%, and $20.2 \%$ were diagnosed with no, one, and $\geq 2$ STIs, respectively. 1,127 $(38.7 \%)$ reported being STI-condom-users. In multivariate analysis, there was no difference in mean STIs diagnosed in the previous year $(0.7$ vs $0.8, p=0.244)$. STI-condom-users were younger ( 39 vs 41 years, $\mathrm{p}=0.004$ ), less likely to live in a suburb with $\geq 10 \%$ gay men $(24.8 \%$ vs $35.5 \%$, p $<0.001)$, less likely to report any condomless sex in the previous 6 months $(85.9 \%$ vs $93.3 \%, \mathrm{p}<0.001)$, and had been on-study less time (1.4 vs 1.6 years, $\mathrm{p}=0.003)$. STI-condom-users were more: concerned about acquiring STIs $(95.1 \%$ vs $88.9 \%$, $\mathrm{p}=0.025)$, likely to discuss STI results with partners $(88.3 \%$ vs $79.8 \%, \mathrm{p}<0.001)$, and likely to believe condoms prevent STI transmission $(96.7 \%$ vs $89.6 \%, \mathrm{p}<0.001)$. Fewer STI-condom-users had rejected a sexual partner for wanting to use condoms $(9.6 \%$ vs $32.7 \%, \mathrm{p}<0.001)$, while more had been rejected for wanting to use condoms $(45.0 \%$ vs $26.2 \%$, $\mathrm{p}<0.001)$.

Conclusion Most PrEP-users did not use condoms for STI prevention, despite strong beliefs in its effectiveness. STI-condomusers did not have fewer STIs, reflecting high levels of condomless sex. Our results suggest alternative methods of STI prevention should be trialled for PrEP-users.

Disclosure No significant relationships. 